\title{
On Euclidean designs and potential energy
}

\author{
Tsuyoshi Miezaki* \\ Department of Mathematics \\ Oita National College of Technology \\ Oita, 870-0152, Japan \\ miezaki@oita-ct.ac.jp \\ Makoto Tagami \\ Department of Mechanical and Control Engineering \\ Niigata Institute of Technology \\ Niigata, 945-1195, Japan \\ tagami@niit.ac.jp
}

Submitted: Jan 17, 2011; Accepted: Dec 14, 2011; Published: Jan 6, 2012

Mathematics Subject Classifications: 05B30, 31C20

\begin{abstract}
We study Euclidean designs from the viewpoint of the potential energy. For a finite set in Euclidean space, we formulate a linear programming bound for the potential energy by applying harmonic analysis on a sphere. We also introduce the concept of strong Euclidean designs from the viewpoint of the linear programming bound, and we give a Fisher type inequality for strong Euclidean designs. A finite set on Euclidean space is called a Euclidean $a$-code if any distinct two points in the set are separated at least by $a$. As a corollary of the linear programming bound, we give a method to determine an upper bound on the cardinalities of Euclidean $a$-codes on concentric spheres of given radii. Similarly we also give a method to determine a lower bound on the cardinalities of Euclidean $t$-designs as an analogue of the linear programming bound.
\end{abstract}

\section{Introduction}

The concept of Euclidean designs is well-known as a natural generalization of spherical designs to Euclidean space. The purpose of this paper is firstly to characterize Euclidean designs in terms of the potential energy, secondly to extend the linear programming bounds on a sphere to configurations of points in Euclidean space, and thirdly to introduce

${ }^{*}$ This work was supported by JSPS KAKENHI. 
the concept of strong Euclidean designs which seems to be natural from the view point of the linear programming bound.

Let $t$ be a natural number, $S^{d-1}$ the $(d-1)$-dimensional unit sphere centered at the origin, and $P_{l}\left(\mathbb{R}^{d}\right)$ the vector space of polynomials of degree at most $l$ in $d$ variables over $\mathbb{R}$. A finite nonempty subset $X$ on $S^{d-1}$ is called a spherical t-design if, for any $f(x) \in P_{t}\left(\mathbb{R}^{d}\right)$, the following equality holds:

$$
\frac{1}{\sigma\left(S^{d-1}\right)} \int_{S^{d-1}} f(x) d \sigma(x)=\frac{1}{|X|} \sum_{x \in X} f(x) .
$$

Here $\sigma$ is an $O\left(\mathbb{R}^{d}\right)$-invariant measure on $S^{d-1}$ and $\sigma\left(S^{d-1}\right)$ denotes the surface volume of the sphere $S^{d-1}$. The concept of spherical designs was defined by Delsarte-Goethals-Seidel [8]. A spherical $t$-design is a configuration of points on the sphere so that the average value of the integral of any polynomial of degree up to $t$ on the sphere is replaced by the average value on the configuration. A finite non-empty subset $X$ on $S^{d-1}(r)$, the sphere of radius $r$ centered at the origin, is also called a spherical $t$-design if $\frac{1}{r} X$ is a spherical $t$-design on the unit sphere $S^{d-1}$.

Let $\mathbb{Z}_{\geqslant 0}, \mathbb{Z}_{>0}$ and $\mathbb{R}_{>0}$ denote the set of non-negative integers, positive integers and positive real numbers, respectively. A spherical $t$-design is closely related to the following Sidelnikov inequality (refer to [14]): for a finite subset $X$ on $S^{d-1}$ and any $l \in \mathbb{Z}_{\geqslant 0}$, it holds that

$$
\frac{1}{|X|^{2}} \sum_{x, y \in X}(x \cdot y)^{l} \geqslant A_{l}:= \begin{cases}\frac{(l-1) ! !(d-2) ! !}{(d+l-2) ! !} & \text { if } l \equiv 0 \quad(\bmod 2) \\ 0 & \text { otherwise }\end{cases}
$$

Here $x \cdot y$ is the standard inner product, and $l ! !:=l(l-2) \cdots$, multiplying down to 1 if $l$ is odd and 2 if $l$ is even, and $0 ! !:=1$. It is well-known that $X$ is a spherical $t$-design if and only if for $0 \leqslant l \leqslant t$, equality holds in (1).

Consider a function $f:(0,4] \rightarrow \mathbb{R}$. Then the potential energy of $X$ for $f$ is defined to be $P E_{f}(X):=\sum_{x \neq y \in X} f\left(\|x-y\|^{2}\right.$ ) (refer to [15, 5]). From Sidelnikov's inequality and the equivalent condition, we see that, for $f_{t}(r)=(4-r)^{t}$, a spherical $t$-design $X$ minimizes the potential energy $P E_{f_{t}}(X)$ among all configurations of $|X|$ points on $S^{d-1}$. It is because

$$
\begin{aligned}
P E_{f_{t}}(X) & =\sum_{x \neq y \in X} f_{t}\left(\|x-y\|^{2}\right) \\
& =\sum_{x \neq y \in X}(2+2(x \cdot y))^{t} \\
& =\sum_{i=0}^{t} a_{i} \sum_{x \neq y \in X}(x \cdot y)^{i},
\end{aligned}
$$

where $a_{i}$ is a positive number. 
The concept of spherical designs was naturally generalized to Euclidean space by Neumaier-Seidel [11]. Let $X$ be a finite subset in $\mathbb{R}^{d}$ and suppose $O \notin X$ (for simplicity, we always suppose this in this paper). We set $R X:=\{\|x\| \mid x \in X\}=\left\{r_{1}, r_{2}, \ldots, r_{p}\right\}$, $S_{i}=r_{i} S^{d-1}, R S:=S_{1} \cup S_{2} \cup \cdots \cup S_{p}$ and $X_{i}=X_{\left(r_{i}\right)}=X \cap S_{i} . \sigma_{i}$ denotes an $O\left(\mathbb{R}^{d}\right)$ invariant measure on $S_{i}$. Also let $w: X \rightarrow \mathbb{R}_{>0}$ and we put $w\left(X_{i}\right):=\sum_{x \in X_{i}} w(x)$.

Definition 1.1 (Neumaier-Seidel [11]). Under the above notation, $(X, w)$ is a Euclidean $t$-design if for any $f(x) \in P_{t}\left(\mathbb{R}^{d}\right)$, the following equality holds:

$$
\sum_{i=1}^{p} \frac{w\left(X_{i}\right)}{\sigma\left(S_{i}\right)} \int_{S_{i}} f(x) d \sigma_{i}(x)=\sum_{x \in X} w(x) f(x) .
$$

In Euclidean space, the following generalized Sidelnikov inequality holds:

Theorem 1 (Neumaier-Seidel [11]). Let $X \subset \mathbb{R}^{d}(|X|<\infty), w: X \rightarrow \mathbb{R}$ and $l \in \mathbb{R}_{\geqslant 0}$. Then the following inequality holds:

$$
\sum_{x, y \in X} w(x) w(y)(x \cdot y)^{l} \geqslant A_{l}\left(\sum_{x \in X} w(x)\|x\|^{l}\right)^{2} .
$$

The actual generalized Sidelnikov inequality given by Neumaier-Seidel [11] is a more general one, but in this paper we write the inequality in the form of Theorem 1 for the purpose of viewing a relation to Euclidean designs.

Let $w^{\prime}: X \longrightarrow \mathbb{R}_{>0}$ and set $w(x)=w^{\prime}(x)\|x\|^{2 j}$ in Theorem 1. Then

$$
\sum_{x, y \in X} w^{\prime}(x) w^{\prime}(y)(\|x\|\|y\|)^{2 j}(x \cdot y)^{l} \geqslant A_{l}\left(\sum_{x \in X} w^{\prime}(x)\|x\|^{2 j+l}\right)^{2} .
$$

$\left(X, w^{\prime}\right)$ is a Euclidean $t$-design if and only if equality holds in $(3)$ for all $j \geqslant 0, l \geqslant 0$ with $2 j+l \leqslant t$ (cf. Lemma 2.5, the proof of Theorem 1 in Section 2).

For $x \in \mathbb{R}^{d} \backslash\{O\}$, we set $\hat{x}:=x /\|x\|$. We define the potential energy of a finite set in Euclidean space which is not necessarily restricted to the sphere:

Definition 1.2. Let $X \subset \mathbb{R}^{d} \backslash\{O\}(|X|<\infty), w: X \rightarrow \mathbb{R}$, and $f: \mathbb{R}_{>0}^{2} \times[-1,1] \rightarrow \mathbb{R}$. Then the potential energy of $(X, w)$ for $f$ is defined by

$$
P E_{f}(X, w)=\sum_{x, y \in X, x \neq y} w(x) w(y) f(\|x\|,\|y\|, \hat{x} \cdot \hat{y})
$$

In particular, for the case when $f(r, s, t)=(r s t)^{l}$, the generalized Sidelnikov inequality gives a lower bound on $P E_{f}(X, w)$.

Let $Q_{i}(t)=Q_{i}^{(d)}(t)$ be the Gegenbauer polynomial of degree $i$ corresponding to the sphere $S^{d-1}$, namely, $\left\{Q_{i}(t)\right\}$ are the orthogonal polynomials on the interval $[-1,1]$ with respect to the weight function $\left(1-t^{2}\right)^{(d-3) / 2}$. In this paper, they are nomalized as $Q_{i}(1)=$ $\operatorname{dim} \operatorname{Harm}_{i}\left(\mathbb{R}^{d}\right)$, where $\operatorname{Harm}_{i}\left(\mathbb{R}^{d}\right)$ is defined in Section 2 (see $\left.(7)\right)$. 
In [15], Yudin gave a linear programming bound on the potential energy using harmonic analysis on the sphere. Applying his method, we obtain a lower bound on the potential energy of a finite set on concentric spheres:

Theorem 2. Let $X \subset \mathbb{R}^{d} \backslash\{O\} \quad(|X|<\infty)$ and $w: X \rightarrow \mathbb{R}$, and let $f: \mathbb{R}_{>0}^{2} \times$ $[-1,1] \rightarrow \mathbb{R}, g_{0}: \mathbb{R}_{>0}^{2} \rightarrow \mathbb{R}$, and $g_{i j}: \mathbb{R}_{>0} \rightarrow \mathbb{R}\left(i, j \in \mathbb{Z}_{>0}\right)$. If $h(r, s, t)=g_{0}(r, s)+$ $\sum_{i, j \geqslant 1} g_{i j}(r) g_{i j}(s) Q_{j}(t)$ satisfies the condition that

$$
f(r, s, t) \geqslant h(r, s, t),(r, s \in R X, t \in[-1,1]),
$$

then the following inequality holds:

$$
P E_{f}(X, w) \geqslant \sum_{x, y \in X} w(x) w(y) g_{0}(\|x\|,\|y\|)-\sum_{x \in X} w(x)^{2} h(\|x\|,\|x\|, 1) .
$$

Moreover equality holds in (4) if and only if for any $x, y \in X(x \neq y), f(\|x\|,\|y\|, \hat{x} \cdot \hat{y})=$ $h(\|x\|,\|y\|, \hat{x} \cdot \hat{y})$, and

$$
\sum_{x, y \in X} w(x) w(y) g_{i j}(\|x\|) g_{i j}(\|y\|) Q_{j}(\hat{x} \cdot \hat{y})=0(\forall i \geqslant 1, \forall j \geqslant 1) .
$$

We give a proof of Theorem 2 in Section 2.

Suppose that $R X$ and $\left|X_{(r)}\right|$ are given and suppose that $g_{0}(r, s)$ is a polynomial and each $g_{i j}(r)$ is a monomial. Then seeking $g_{0}$ and $g_{i j}$ which maximize the lower bound (4) in Theorem 2, is reduced to solving a linear programming problem. Therefore we may consider Theorem 2 as a linear programming bound in Euclidean space.

Next we set $g_{i j}(r):=a_{i j} r^{2 i+j}\left(a_{i j} \neq 0\right)$ if $2 i+j \leqslant t$, and $g_{i j}(r):=0$ otherwise. Then we will see by Lemma 2.5 that the condition (5) is equivalent for $(X, w)$ to be a Euclidean $t$-design. Therefore we see that a Euclidean $t$-design minimizes the potential energy for the functions $h(r, s, t)=g_{0}(r, s)+\sum_{i, j \geqslant 1} g_{i j}(r) g_{i j}(s) Q_{j}(t)$. The purpose of this paper is to introduce the following concept:

Definition 1.3. $(X, w)$ is called a strong Euclidean t-design if the following condition holds:

$$
\sum_{x, y \in X} w(x) w(y)(\|x\|\|y\|)^{i} Q_{j}(\hat{x} \cdot \hat{y})=0(0 \leqslant \forall i \leqslant t, 1 \leqslant \forall j \leqslant t) .
$$

Strong Euclidean $t$-designs can be interpreted as the strongest designs among those minimizing the potential energy in the case when we take monomials as $g_{i j}$ 's in Theorem 2. Also the property of being strong Euclidean $t$-designs can be expressed as being finite sets on concentric spheres such that, for any continuous function on some space depending on the parameter $t$, the average value of the integral on the concentric spheres is replaced by the average value on the set (see the proof of Theorem 3 in Section 3). Therefore by Seymour-Zaslavsky's theorem [13], strong Euclidean $t$-designs exist for any $t$.

For Euclidean designs, the Fisher type inequality is famous in algebraic combinatorics and numerical analysis (cf. $[7,9,10,2]$ ). Therefore it is natural to ask whether a Fisher type inequality holds for strong Euclidean designs, too. We say $(X, w)$ is antipodal if $X=-X, w(x)=w(-x)$. Then the following is the main result of this paper: 
Theorem 3. Let $(X, w)$ be a strong Euclidean t-design on $p$ concentric spheres. Assume that $p \geqslant e+1$. Then the following inequality holds:

$$
|X| \geqslant \begin{cases}(e+1)\left\{\left(\begin{array}{c}
d+e-1 \\
e
\end{array}\right)+\left(\begin{array}{c}
d+e-2 \\
e-1
\end{array}\right)\right\} & \text { if } t=2 e \\
2(e+1)\left(\begin{array}{c}
d+e-1 \\
e
\end{array}\right) & \text { if }(X, w) \text { is antipodal and } \\
& t=2 e+1 .\end{cases}
$$

We give a proof of Theorem 3 in Section 3 .

Let $(X, w)$ be a strong Euclidean $t$-design on $p$ concentric spheres and assume $p \leqslant t+1$. Then we will see by Lemma 3.1 that each $X_{i}$ is a spherical $t$-design. On the other hand, it is easy to see that if $X_{i}$ is a spherical $t$-design for any $i$, then $X$ is a strong Euclidean $t$-design. Hence when $p \leqslant t+1$, strong Euclidean $t$-designs are characterized by the property that each $X_{i}$ is a spherical $t$-design. So it is essential to consider the case when $p \geqslant t+2$. Such an example certainly exists. For example, let $X=\{(1,0),(-1,1),(-1,-1),(2,2),(2,-2),(-3,0)\}$ and $w \equiv 1$. Then $(X, w)$ is a strong Euclidean 1-design, but each $X_{i}$ is not a spherical 1-design.

Moreover if a tight spherical $t$-design exists on $S^{d-1}$ (for the definition of tight spherical $t$-design, see [8] or Theorem 4 in Section 3 of this paper), then putting a tight spherical $t$-design on each $e+1$ concentric sphere, we obtain an example attaining the lower bound in Theorem 3.

Definition 1.4. A finite set in Euclidean space is called a Euclidean a-code if any distinct two points in the set are separated at least by $a$. ds.

Finally in Section 4, as a corollary of Theorem 2, we give a method to calculate an upper bound of the cardinality of a Euclidean $a$-code under the condition that the radii of concentric spheres on which the code lies are given.

\section{Linear programming bound}

Let $\triangle$ be the Laplacian, that is, $\triangle=\frac{\partial^{2}}{\partial x_{1}^{2}}+\cdots+\frac{\partial^{2}}{\partial x_{d}^{2}}$, and set

$$
\begin{aligned}
\operatorname{Hom}_{i}\left(\mathbb{R}^{d}\right) & =\left\{f(x) \in \mathbb{R}\left[x_{1}, \ldots, x_{d}\right] \mid f(x) \text { is homogeneous of degree } i\right\}, \\
\operatorname{Harm}_{i}\left(\mathbb{R}^{d}\right) & =\left\{f(x) \in \operatorname{Hom}_{i}\left(\mathbb{R}^{d}\right) \mid \triangle(f(x))=0\right\}
\end{aligned}
$$

First we give an equivalent condition for $X$ to be a spherical $t$-design:

Lemma 2.1 (cf. [8]). Let $X$ be a finite non-empty set on $S^{d-1}$. Then the following are equivalent:

(1) $X$ is a spherical t-design. 
(2) For any $1 \leqslant l \leqslant t, \phi \in \operatorname{Harm}_{l}\left(\mathbb{R}^{d}\right)$,

$$
\sum_{x \in X} \phi(x)=0
$$

We also give an equivalent condition for $(X, w)$ to be a Euclidean $t$-design:

Lemma 2.2 (cf. [11]). Let $X$ be a finite non-empty set in $\mathbb{R}^{d} \backslash\{0\}$ and $w: X \rightarrow \mathbb{R}_{>0}$. Then the following are equivalent:

(1) $(X, w)$ is a Euclidean t-design.

(2) For any $1 \leqslant l \leqslant t, \phi \in \operatorname{Harm}_{l}\left(\mathbb{R}^{d}\right)$ and $0 \leqslant j \leqslant\left[\frac{t-l}{2}\right]$,

$$
\sum_{x \in X} w(x)\|x\|^{2 j} \phi(x)=0
$$

We define a non-degenerate inner product in the space $P_{l}\left(\mathbb{R}^{d}\right)$ as follows: for $f, g \in$ $P_{l}\left(\mathbb{R}^{d}\right)$

$$
\langle f, g\rangle:=\int_{S^{d-1}} f(x) g(x) d \sigma(x) .
$$

In a similar way to the above, define an inner product in the vector spaces $\operatorname{Hom}_{i}\left(\mathbb{R}^{d}\right)$ and $\operatorname{Harm}_{i}\left(\mathbb{R}^{d}\right)$. The following addition formula of the Gegenbauer polynomials is well-known:

Lemma 2.3 (cf. [8]). Let $\left\{\phi_{l, 1}, \ldots \phi_{l, h_{l}}\right\}$ be an orthonormal basis of $\operatorname{Harm}_{l}\left(\mathbb{R}^{d}\right)$. Then for any $x, y \in S^{d-1}$, we have

$$
\sum_{i=1}^{h_{l}} \phi_{l, i}(x) \phi_{l, i}(y)=Q_{l}(x \cdot y) .
$$

The following is immediate from the previous lemma.

Lemma 2.4 (cf. [12]). For any non-negative integer $i$ and any finite subset $X \subset S^{d-1}$, the matrix $\left(Q_{i}(x \cdot y)\right)_{x, y \in X}$ indexed by $X \times X$ is positive semi-definite.

We state an equivalent condition for $(X, w)$ to be a Euclidean $t$-design, in terms of the Gegenbauer polynomials.

Lemma 2.5. $(X, w)$ is a Euclidean $t$-design if and only if for $1 \leqslant l \leqslant t$ and $0 \leqslant j \leqslant\left[\frac{t-l}{2}\right]$, the following equality holds:

$$
\sum_{x, y \in X} w(x) w(y)(\|x\|\|y\|)^{l+2 j} Q_{l}(\hat{x} \cdot \hat{y})=0 .
$$


Proof. Let $\left\{\phi_{l, 1}, \ldots \phi_{l, h_{l}}\right\}$ be an orthonormal basis of $\operatorname{Harm}_{l}\left(\mathbb{R}^{d}\right)$. Then

$$
\begin{aligned}
\sum_{i=1}^{h_{l}}\left(\sum_{x \in X} w(x)\|x\|^{2 j} \phi_{l, i}(x)\right)^{2} & =\sum_{i=1}^{h_{l}} \sum_{x, y \in X} w(x) w(y)(\|x\|\|y\|)^{2 j} \phi_{l, i}(x) \phi_{l, i}(y) \\
& =\sum_{i=1}^{h_{l}} \sum_{x, y \in X} w(x) w(y)(\|x\|\|y\|)^{2 j+l} \phi_{l, i}(\hat{x}) \phi_{l, i}(\hat{y}) \\
& =\sum_{x, y \in X} w(x) w(y)(\|x\|\|y\|)^{2 j+l} Q_{l}(\hat{x} \cdot \hat{y}) .
\end{aligned}
$$

The last equality follows from Lemma 2.3. By Lemma 2.2, $X$ is a Euclidean $t$-design if and only if $\sum_{i=1}^{h_{l}}\left(\sum_{x \in X} w(x)\|x\|^{2 j} \phi_{l, i}(x)\right)^{2}=0$ holds for $1 \leqslant i \leqslant h_{l}, 1 \leqslant l \leqslant t$ and $0 \leqslant j \leqslant\left[\frac{t-l}{2}\right]$. Therefore the proof is completed.

Proof of Theorem 2. By Definition 1.2,

$$
P E_{f}(X)=\sum_{x, y \in X, x \neq y} w(x) w(y) f(\|x\|,\|y\|, \hat{x} \cdot \hat{y}) .
$$

Since $f(r, s, t) \geqslant h(r, s, t)$ for any $r, s$ and $t$ and since $w(x)>0$ for any $x \in X$, we have

$$
\begin{aligned}
P E_{f}(X) & \geqslant \sum_{x \neq y \in X} w(x) w(y) h(\|x\|,\|y\|, \hat{x} \cdot \hat{y}) \\
& =\sum_{x, y \in X} w(x) w(y) h(\|x\|,\|y\|, \hat{x} \cdot \hat{y})-\sum_{x \in X} w(x)^{2} h(\|x\|,\|x\|, 1) \\
& =\sum_{x, y \in X} w(x) w(y) g_{0}(\|x\|,\|y\|) \\
& +\sum_{x, y \in X} \sum_{i, j \geqslant 1} w(x) w(y) g_{i j}(\|x\|) g_{i j}(\|y\|) Q_{j}(\hat{x} \cdot \hat{y}) \\
& -\sum_{x \in X} w(x)^{2} h(\|x\|,\|x\|, 1) .
\end{aligned}
$$

By Lemma $2.4,\left(Q_{j}(x \cdot y)\right)$ is positive semi-definite. Hence for any $i, j \geqslant 1$, we have

$$
\sum_{x, y \in X} w(x) w(y) g_{i j}(\|x\|) g_{i j}(\|y\|) Q_{j}(\hat{x} \cdot \hat{y}) \geqslant 0 .
$$

Namely

$$
P E_{f}(X) \geqslant \sum_{x, y \in X} w(x) w(y) g_{0}(\|x\|,\|y\|)-\sum_{x \in X} w(x)^{2} h(\|x\|,\|x\|, 1) .
$$

Therefore the inequality (4) holds. The condition to satisfy equality in (4) is clear. 
Now by using Theorem 2, it is easy to prove the generalized Sidelnikov inequality in Theorem 1. First we quote the following well-known lemma:

Lemma 2.6 ([1], Lemma 3.4.3). Let $t^{l}=\sum_{i=0}^{l} A_{l, i} Q_{l-i}(t)$ be the expansion in the Gegenbauer polynomials. Then

$$
A_{l, i}= \begin{cases}\frac{l ! !(d-2) ! !}{i ! !(d+2 l-i-2) ! !} & \text { if } i \equiv 0 \quad(\bmod 2), \\ 0 & \text { otherwise. }\end{cases}
$$

Proof of Theorem 1. In Theorem 2, we set $f(r, s, t)=(r s)^{l} t^{l}$,

$$
\begin{cases}g_{0 j}(r)=\sqrt{A_{l, l-j}} r^{l} & 0 \leqslant j \leqslant l \\ g_{i j}(r)=0 & i \neq 0\end{cases}
$$

and $g_{0}(r, s)=g_{00}(r) g_{00}(s)$. Then by Lemma 2.6,

$$
\begin{aligned}
h(r, s, t) & =g_{0}(r, s)+\sum_{i, j \geqslant 1} g_{i j}(r) g_{i j}(s) Q_{j}(t) \\
& =\sum_{j=0}^{l} A_{l, l-j}(r s)^{l} Q_{j}(t)=(r s)^{l} t^{l}=f(r, s, t) .
\end{aligned}
$$

Therefore the conditions in Theorem 2 hold. Hence

$$
\begin{aligned}
P E_{f}(X) & =\sum_{x \neq y \in X} w(x) w(y) f(\|x\|,\|y\|, \hat{x} \cdot \hat{y})=\sum_{x \neq y \in X} w(x) w(y)(x \cdot y)^{l} \\
& \geqslant \sum_{x, y \in X} w(x) w(y) g_{0}(\|x\|,\|y\|)-\sum_{x \in X} w(x)^{2} h(\|x\|,\|x\|, 1) \\
& =\sum_{x, y \in X} w(x) w(y) A_{l, l}(\|x\|\|y\|)^{l}-\sum_{x \in X} w(x)^{2} f(\|x\|,\|x\|, 1) \\
& =A_{l, l}\left(\sum_{x \in X} w(x)\|x\|^{l}\right)^{2}-\sum_{x \in X} w(x)^{2} f(\|x\|,\|x\|, 1) .
\end{aligned}
$$

Since $A_{l, l}=A_{l}$, we have

$$
\sum_{x, y \in X} w(x) w(y)(x \cdot y)^{l} \geqslant A_{l}\left(\sum_{x \in X} w(x)\|x\|^{l}\right)^{2} .
$$

\section{$3 \quad$ Fisher type inequality}

In this section, we give a Fisher type inequality for strong Euclidean designs. First we show the following lemma: 
Lemma 3.1. Let $(X, w)$ be a strong Euclidean t-design on $p$ concentric spheres, and $w: X \rightarrow \mathbb{R}_{>0}$ be constant on each concentric sphere. Suppose $t+1 \geqslant p$. Then each $X_{i}$ is a spherical t-design.

Proof. Let $(X, w)$ be a strong Euclidean $t$-design. Then by Definition 1.3 , for $0 \leqslant i \leqslant t$ and $1 \leqslant j \leqslant t$

$$
\sum_{x, y \in X} w(x) w(y)(\|x\|\|y\|)^{i} Q_{j}(\hat{x} \cdot \hat{y})=0
$$

Let $\left\{\phi_{l, 1}, \ldots \phi_{l, h_{l}}\right\}$ be an orthonormal basis of $\operatorname{Harm}_{l}\left(\mathbb{R}^{d}\right)$. Then for any $j$,

$$
\begin{aligned}
\sum_{i=1}^{h_{l}}\left(\sum_{x \in X} w(x)\|x\|^{j} \phi_{l, i}(x)\right)^{2} & =\sum_{i=1}^{h_{l}} \sum_{x, y \in X} w(x) w(y)(\|x\|\|y\|)^{j} \phi_{l, i}(x) \phi_{l, i}(y) \\
& =\sum_{i=1}^{h_{l}} \sum_{x, y \in X} w(x) w(y)(\|x\|\|y\|)^{j+l} \phi_{l, i}(\hat{x}) \phi_{l, i}(\hat{y}) \\
& =\sum_{x, y \in X} w(x) w(y)(\|x\|\|y\|)^{j+l} Q_{l}(\hat{x} \cdot \hat{y}) .
\end{aligned}
$$

The last equality follows from Lemma 2.3. Therefore $(X, w)$ is a strong Euclidean $t$ design if and only if the following equalities hold: for $1 \leqslant l \leqslant t, \phi(x) \in \operatorname{Harm}_{l}\left(\mathbb{R}^{d}\right)$ and $-l \leqslant j \leqslant t-l$,

$$
\sum_{x \in X} w(x)\|x\|^{j} \phi(x)=\sum_{i=1}^{p}\left|r_{i}\right|^{j} \sum_{x \in X_{i}} w(x) \phi(x)=0 .
$$

Fix $\phi \in \operatorname{Harm}_{l}\left(\mathbb{R}^{d}\right)$ and regard $\left\{\sum_{x \in X_{i}} w(x) \phi(x)\right\}_{i=1}^{p}$ as variables. Then the matrix coefficient of the linear system (8) is

$$
\left(\begin{array}{cccc}
r_{1}^{-l} & r_{2}^{-l} & \ldots & r_{p}^{-l} \\
r_{1}^{-l+1} & r_{2}^{-l+1} & \ldots & r_{p}^{-l+1} \\
\vdots & \vdots & \vdots & \vdots \\
r_{1}^{t-l} & r_{2}^{t-l} & \ldots & r_{p}^{t-l}
\end{array}\right)
$$

When $t+1 \geqslant p$, the rank of this matrix is $p$. Hence for any $1 \leqslant i \leqslant p$ and $\phi \in \operatorname{Harm}_{l}\left(\mathbb{R}^{d}\right)$, we have

$$
\sum_{x \in X_{i}} w(x) \phi(x)=0
$$

Now because of the fact that $w(x)$ is constant on each concentric sphere, each $X_{i}$ is a spherical $t$-design by Lemma 2.1 .

The following theorem is the well-known Fisher type inequality for spherical designs: 
Theorem 4 (Delsarte-Goethals-Seidel [8]). Let $X \subset S^{d-1}$ be a spherical t-design. Then

$$
|X| \geqslant \begin{cases}\left(\begin{array}{c}
d+e-1 \\
e
\end{array}\right)+\left(\begin{array}{c}
d+e-2 \\
e-1
\end{array}\right) & \text { if } t=2 e, \\
2\left(\begin{array}{c}
d+e-1 \\
e
\end{array}\right) & \text { if } t=2 e+1 .\end{cases}
$$

A spherical $t$-design $X$ is tight if equality holds in (9). By Lemma 3.1 and Theorem 4, we obtain the following corollary:

Corollary 3.1. Let $(X, w)$ be a strong Euclidean t-design on p concentric spheres. Suppose that $w: X \rightarrow \mathbb{R}_{>0}$ be constant on each concentric sphere and that $t+1 \geqslant p$. Then

$$
|X| \geqslant \begin{cases}p\left\{\left(\begin{array}{c}
d+e-1 \\
e
\end{array}\right)+\left(\begin{array}{c}
d+e-2 \\
e-1
\end{array}\right)\right\} & \text { if } t=2 e \\
2 p\left(\begin{array}{c}
d+e-1 \\
e
\end{array}\right) & \text { if } t=2 e+1 .\end{cases}
$$

In the sequel, suppose that $p$ is sufficiently large comparing to $t$. Our proof below follows Delsarte-Seidel [7]. For a subspace $P$ of $\operatorname{Pol}\left(\mathbb{R}^{d}\right)$, put $\|x\|^{j} P:=\left\{\|x\|^{j} f(x) \mid f \in\right.$ $P\}$. We set

$$
\operatorname{Pol}^{\prime}\left(\mathbb{R}^{d}\right):=\operatorname{Pol}\left(\mathbb{R}^{d}\right)+\|x\| \operatorname{Pol}\left(\mathbb{R}^{d}\right) .
$$

We remark that the sum of the right hand side is a direct sum. It is because, if there exist nonzeros $f, g \in \operatorname{Pol}\left(\mathbb{R}^{d}\right)$ such that $f+\|x\| g=0$, then $f^{2}=\|x\|^{2} g^{2}$. Because $\|x\|^{2}=x_{1}^{2}+\cdots+x_{d}^{2}$ is irreducible in $\operatorname{Pol}\left(\mathbb{R}^{d}\right)$, we have a contradiction since the parities of $\|x\|^{2}=x_{1}^{2}+\cdots+x_{d}^{2}$ in the left and right hand side are different. Set

$$
\begin{aligned}
\operatorname{Pol}_{j}^{\prime}\left(\mathbb{R}_{d}\right) & :=\operatorname{Pol}_{j}\left(\mathbb{R}_{d}\right)+\|x\| \operatorname{Pol}_{j-1}\left(\mathbb{R}_{d}\right), \\
\operatorname{Hom}_{j}^{\prime}\left(\mathbb{R}^{d}\right) & :=\operatorname{Hom}_{j}\left(\mathbb{R}^{d}\right)+\|x\| \operatorname{Hom}_{j-1}\left(\mathbb{R}^{d}\right) .
\end{aligned}
$$

Then

$$
\operatorname{Pol}_{j}^{\prime}\left(\mathbb{R}^{d}\right)=\bigoplus_{i=0}^{j} \operatorname{Hom}_{j}^{\prime}\left(\mathbb{R}^{d}\right) .
$$

Generally for $T \subset \mathbb{R}^{d}$, we denote by $\operatorname{Hom}_{l}(T)$ (resp. $\operatorname{Harm}_{l}(T)$ ) the vector space of elements of $\operatorname{Hom}_{l}\left(\mathbb{R}^{d}\right)$ (resp. $\left.\operatorname{Harm}_{l}\left(\mathbb{R}^{d}\right)\right)$ which are restricted on $T$. For example we write $\operatorname{Pol}(T)=\left\{\left.f\right|_{T} \mid f \in \operatorname{Pol}\left(\mathbb{R}^{d}\right)\right\}$, where $\left.f\right|_{T}$ denotes a restricted function on $T$ for $f$.

\section{Lemma 3.2.}

$$
\operatorname{Hom}_{j}^{\prime}(R S)=\operatorname{Hom}_{j}(R S) \oplus\left(\|x\| \operatorname{Hom}_{j-1}\right)(R S) .
$$

Proof. Take any $f \in \operatorname{Hom}_{j}(R S)$ and $g \in \operatorname{Hom}_{j-1}(R S)$ such that $f=\|x\| g$, then we have $f^{2}(x)=\|x\|^{2} g^{2}(x)$ as polynomials. Since $\|x\|^{2}=x_{1}^{2}+x_{2}^{2}+\cdots+x_{d}^{2}$ is an irreducible element of the polynomial ring, checking the parities of $\|x\|^{2}=x_{1}^{2}+x_{2}^{2}+\cdots+x_{d}^{2}$ in the left and right hand side, we have $f=g=0$. Therefore the sum of the right hand side is a direct sum. 
Lemma 3.3. Suppose that $R S$ consists of $p$ concentric spheres. Then we have

$$
\operatorname{Hom}_{j}^{\prime}(R S) \subset \sum_{i=1}^{p} \operatorname{Hom}_{j+i}^{\prime}(R S) .
$$

Proof. For $f \in \operatorname{Hom}_{j}^{\prime}\left(\mathbb{R}^{d}\right)$, we have the following identity on $R S$ : for $y \in R S$

$$
f(y) \prod_{r \in R X}(r-\|y\|)=0 .
$$

Expanding (11), we see that $f(y)$ is written as a linear combination with respect to $\|y\|^{i} f(y)(i=1,2, \ldots, p)$, where $\|y\|^{i} f(y) \in \operatorname{Hom}_{j+i}^{\prime}(R S)$.

Lemma 3.4. Suppose that $R S$ consists of $p$ concentric spheres. Then we have

$$
\mathrm{Pol}_{j}^{\prime}(R S)=\bigoplus_{i=0}^{p-1} \operatorname{Hom}_{j-i}^{\prime}(R S)
$$

Proof. By (10), we have

$$
\operatorname{Pol}_{j}^{\prime}(R S)=\sum_{i=0}^{j} \operatorname{Hom}_{i}^{\prime}(R S)=\sum_{i=0}^{p-1} \operatorname{Hom}_{j-i}^{\prime}(R S) .
$$

Therefore it is enough to show that the sum of the right hand side is a direct sum. First we show that for the restriction homomorphism $\phi: \operatorname{Pol}_{j}^{\prime}\left(\mathbb{R}^{d}\right) \rightarrow \operatorname{Pol}_{j}^{\prime}(R S)$,

$$
\operatorname{Ker} \phi=\operatorname{Pol}_{j-p}^{\prime}\left(\mathbb{R}^{d}\right) \prod_{r \in R X}(r-\|x\|) \text {. }
$$

Clearly we have

$$
\operatorname{Ker} \phi \supset \operatorname{Pol}_{j-p}^{\prime}\left(\mathbb{R}^{d}\right) \prod_{r \in R X}(r-\|x\|) .
$$

Conversely, take $f+\|x\| g \in \operatorname{Ker} \phi,\left(f \in \operatorname{Pol}_{j}\left(\mathbb{R}^{d}\right), g \in \operatorname{Pol}_{j-1}\left(\mathbb{R}^{d}\right)\right)$. For $r_{1} \in R X$,

$$
f(x)+\|x\| g(x)=f(x)+r_{1} g(x)-\left(r_{1}-\|x\|\right) g(x) .
$$

Hence $f(x)+r_{1} g(x)$ is zero on $r_{1} S^{d-1}$. By Hilbert's Nullstellensatz, there exists some $h(x) \in \operatorname{Pol}_{j-2}\left(\mathbb{R}^{d}\right)$ such that $f(x)+r_{1} g(x)=\left(r_{1}^{2}-\|x\|^{2}\right) h(x)$. Therefore we have

$$
f(x)+\|x\| g(x)=\left(r_{1}-\|x\|\right)\left\{\left(r_{1}+\|x\|\right) h(x)-g(x)\right\} .
$$

Similarly, replacing $f(x)+\|x\| g(x)$ and $r_{1}$ by $\left(r_{1}+\|x\|\right) h(x)-g(x)$ and $r_{2}$, respectively, we see that there exists $q(x) \in \mathrm{Pol}_{j-2}^{\prime}\left(\mathbb{R}^{d}\right)$ such that

$$
f(x)+\|x\| g(x)=\left(r_{1}-\|x\|\right)\left(r_{2}-\|x\|\right) q(x) .
$$


Recursively we see that there exists $r(x) \in \operatorname{Pol}_{j-p}^{\prime}\left(\mathbb{R}^{d}\right)$ such that

$$
f(x)+\|x\| g(x)=\prod_{r \in R X}(r-\|x\|) r(x) .
$$

Therefore we have $\operatorname{Ker} \phi \subset \operatorname{Pol}_{j-p}^{\prime}\left(\mathbb{R}^{d}\right) \prod_{r \in R X}(r-\|x\|)$.

By (13),

$$
\operatorname{dim} \operatorname{Pol}_{j}^{\prime}(R S)=\operatorname{dim} \operatorname{Pol}_{j}^{\prime}\left(\mathbb{R}^{d}\right)-\operatorname{dim} \operatorname{Pol}_{j-p}^{\prime}\left(\mathbb{R}^{d}\right) .
$$

Using $\operatorname{Hom}_{i}^{\prime}\left(\mathbb{R}^{d}\right) \simeq \operatorname{Hom}_{i}^{\prime}(R S)$, we have

$$
\operatorname{dim} \operatorname{Pol}_{j}^{\prime}(R S)=\sum_{i=0}^{p-1} \operatorname{dim} \operatorname{Hom}_{j-i}^{\prime}\left(\mathbb{R}^{d}\right)=\sum_{i=0}^{p-1} \operatorname{dim} \operatorname{Hom}_{j-i}^{\prime}(R S) .
$$

This implies that the sum of the right hand side in (12) is a direct sum.

Proof of Theorem 3. The following decomposition is well-known (cf. [11]):

$$
\operatorname{Hom}_{i}\left(\mathbb{R}^{d}\right)=\bigoplus_{j=0}^{\left[\frac{i}{2}\right]}\|x\|^{2 j} \operatorname{Harm}_{i-2 j}\left(\mathbb{R}^{d}\right)
$$

We set

$$
P_{t}:=\bigoplus_{i=0}^{t} \bigoplus_{j=-i}^{t-i}\|x\|^{j} \operatorname{Harm}_{i}\left(\mathbb{R}^{d}\right)=\sum_{i=0}^{t} \sum_{j=-i}^{t-i}\|x\|^{j} \operatorname{Hom}_{i}\left(\mathbb{R}^{d}\right) .
$$

Then for any $f(x)=\sum_{i=0}^{t} \sum_{j=-i}^{t-i}\|x\|^{j} f_{i}(x) \in P_{t}$ with $f_{i} \in \operatorname{Harm}_{i}\left(\mathbb{R}^{d}\right)$,

$$
\begin{aligned}
& \sum_{k=1}^{p} \frac{w\left(X_{k}\right)}{\left|S_{k}\right|} \int_{S_{k}} f(x) d \sigma_{k}(x)=\sum_{k=1}^{p} \sum_{j=-i}^{t-i} r_{k}^{j} \frac{w\left(X_{k}\right)}{\left|S_{k}\right|} \sum_{i=0}^{t} \int_{S_{k}} f_{i}(x) d \sigma_{k}(x) \\
= & \sum_{k=1}^{p} \sum_{j=-i}^{t-i} r_{k}^{j} \frac{w\left(X_{k}\right)}{\left|S_{k}\right|} \int_{S_{k}} f_{0}(x) d \sigma_{k}(x)=\sum_{k=1}^{p} \sum_{j=-i}^{t-i} r_{k}^{j} w\left(X_{k}\right) f_{0}(x) \\
= & \sum_{k=1}^{p} \sum_{j=-i}^{t-i} \sum_{x \in X_{k}} w(x)\|x\|^{j} f_{0}(x)=\sum_{j=-i}^{t-i} \sum_{x \in X} w(x)\|x\|^{j} f_{0}(x) .
\end{aligned}
$$

Let $(X, w)$ be a strong Euclidean $t$-design. Then by the equivalent condition $(8)$ for $(X, w)$ to be a strong Euclidean $t$-design, we have

$$
\sum_{j=-i}^{t-i} \sum_{x \in X} w(x)\|x\|^{j} f_{0}(x)=\sum_{i=0}^{t} \sum_{j=-i}^{t-i} \sum_{x \in X} w(x)\|x\|^{j} f_{i}(x)=\sum_{x \in X} w(x) f(x) .
$$

Therefore for any $f \in P_{t}$,

$$
\sum_{k=1}^{p} \frac{w\left(X_{k}\right)}{\left|S_{k}\right|} \int_{S_{k}} f(x) d \sigma_{k}(x)=\sum_{x \in X} w(x) f(x) .
$$


Suppose $t=2 e$. Then we have $P_{t}=P_{e} P_{e}=\left\langle f \cdot g \mid f, g \in P_{e}\right\rangle$, where $\left\langle f \cdot g \mid f, g \in P_{e}\right\rangle$ is the vector space expanded by $f \cdot g\left(f, g \in P_{e}\right)$.

We define the non-degenerate inner products $[\cdot, \cdot]$ and $\langle\cdot, \cdot\rangle_{R S}$ on $P_{e}(X)$ and $P_{e}(R S)$, respectively, as follows: for $f, g \in P_{e}$,

$$
\begin{aligned}
{[f, g] } & :=\sum_{x \in X} w(x) f(x) g(x) \\
\langle f, g\rangle_{R S} & :=\sum_{i=1}^{p} \frac{w\left(X_{i}\right)}{\left|S_{i}\right|} \int_{S_{i}} f(x) g(x) d \sigma_{i}(x) .
\end{aligned}
$$

Then (14) is equivalent to that, for any $f, g \in P_{e}$,

$$
[f, g]=\langle f, g\rangle_{R S}
$$

This implies that the restriction map $\rho_{e}: P_{e}(R S) \rightarrow P_{e}(X)$ is an injective homomorphism. Hence $|X|$ is bounded below by $\operatorname{dim} P_{e}(R S)$.

Set $T_{e}:=\left(\|x\|^{e} P_{e}\right)(R S)=\sum_{i=0}^{t} \sum_{j=-i}^{t-i}\left(\|x\|^{e+j} \operatorname{Hom}_{i}\right)(R S)$. Since $\operatorname{dim} P_{e}(R S)=$ $\operatorname{dim} T_{e}$, it is enough to calculate $\operatorname{dim} T_{e}$. Generally we have the following:

$$
\left(\|x\|^{2} \operatorname{Hom}_{i-2}\right)(R S) \subset \operatorname{Hom}_{i}(R S)
$$

Therefore

$$
T_{e}=\sum_{i=1}^{e+1}\left(\|x\|^{i} \operatorname{Hom}_{e-1}\right)(R S)+\sum_{i=0}^{e}\left(\|x\|^{i} \operatorname{Hom}_{e}\right)(R S) .
$$

By the assumption $p \geqslant e+1$, this sum is a direct sum by Lemma 3.2 and 3.4. So we have

$$
\operatorname{dim} T_{e}=(e+1)\left\{\left(\begin{array}{c}
d+e-1 \\
e
\end{array}\right)+\left(\begin{array}{c}
d+e-2 \\
e-1
\end{array}\right)\right\} .
$$

Next we suppose that $(X, w)$ is antipodal and that $t=2 e+1$. Then set

$$
P_{2 e}^{\prime}\left(\mathbb{R}^{d}\right)=\bigoplus_{i=0}^{e} \bigoplus_{j=-2 i}^{2 e+1-2 i}\|x\|^{j} \operatorname{Harm}_{2 i}\left(\mathbb{R}^{d}\right)=\sum_{i=0}^{e} \sum_{j=-2 i}^{2 e+1-2 i}\|x\|^{j} \operatorname{Hom}_{2 i}\left(\mathbb{R}^{d}\right)
$$

We assume that $X$ is a disjoint union of $Y$ and $-Y$. Then in a similar way to the case when $t=2 e$, we see that $(X, w)$ is a strong Euclidean $(2 e+1)$-design if and only if for any $f \in P_{2 e}^{\prime}$ the following holds:

$$
\sum_{i=1}^{p} \frac{w\left(X_{i}\right)}{\left|S_{i}\right|} \int_{S_{i}} f(x) d \sigma_{i}(x)=\sum_{y \in Y} w(y) f(y) .
$$

Set

$$
P_{e}^{\prime \prime}\left(\mathbb{R}^{d}\right)=\sum_{k=0}^{\left[\frac{e}{2}\right]} \sum_{j=-e+2 k}^{2 k}\|x\|^{j} \operatorname{Hom}_{e-2 k}\left(\mathbb{R}^{d}\right)
$$


Then we have $P_{2 e}^{\prime}\left(\mathbb{R}^{d}\right) \supset P_{e}^{\prime \prime}\left(\mathbb{R}^{d}\right) \cdot P_{e}^{\prime \prime}\left(\mathbb{R}^{d}\right)$. Therefore when we also define the nondegenerate inner products in the space $P_{e}^{\prime \prime}(Y)$ and $P_{e}^{\prime \prime}(R S)$ in the same way as (15) and (16), we see by (18) that, for any $f, g \in P_{e}^{\prime \prime}$

$$
[f, g]=\langle f, g\rangle_{R S} .
$$

Therefore we see that the restriction map $\rho_{e}^{\prime}: P_{e}^{\prime \prime}(R S) \rightarrow P_{e}^{\prime \prime}(Y)$ is injective. Hence, $|Y|$ is bounded below by $\operatorname{dim} P_{e}^{\prime \prime}(R S)$. In particular, $|X|=2|Y| \geqslant 2 \operatorname{dim} P_{e}^{\prime \prime}(R S)$. Set $T_{e}^{\prime}:=\left(\|x\|^{e} P_{e}^{\prime \prime}\right)(R S)$. Then from the fact that $\operatorname{dim} P_{e}^{\prime \prime}(R S)=\operatorname{dim} T_{e}^{\prime}$ and from (17), we have

$$
T_{e}^{\prime}=\operatorname{Hom}_{e}(R S)+\|x\| \operatorname{Hom}_{e}(R S)+\cdots+\|x\|^{e} \operatorname{Hom}_{e}(R S) .
$$

When $p \geqslant e+1$, by Lemma 3.4, the sum of the right hand side in (19) is a direct sum. Therefore $\operatorname{dim} T_{e}^{\prime}=(e+1)\left(\begin{array}{c}d+e-1 \\ e\end{array}\right)$.

\section{Bounds for Euclidean $a$-codes and Euclidean de- signs}

In this section, we give a method to obtain a bound of the cardinality of Euclidean $a$-codes and Euclidean designs.

Theorem 5. Let $X \subset \mathbb{R}^{d}(|X|<\infty)$ be a Euclidean a-code, $g_{0}: \mathbb{R}_{>0}^{2} \rightarrow \mathbb{R}, g_{i j}: \mathbb{R}_{>0} \rightarrow$ $\mathbb{R}(i, j \geqslant 1)$ and $h(r, s, t)=g_{0}(r, s)+\sum_{i, j \geqslant 1} g_{i j}(r) g_{i j}(s) Q_{j}(t)$. Assume that

$$
h(r, s, t) \leqslant 0,\left(r^{2}+s^{2}-2 r s t \geqslant a^{2}, r, s \in R X\right) .
$$

Then we have the following inequality:

$$
\sum_{x, y \in X} g_{0}(\|x\|,\|y\|) \leqslant \sum_{x \in X} h(\|x\|,\|x\|, 1) .
$$

Proof. Set

$$
f(r, s, t)= \begin{cases}+\infty & \text { if } r^{2}+s^{2}-2 r s t<a^{2}, \\ 0 & \text { otherwise. }\end{cases}
$$

Since $h(r, s, t) \leqslant 0,\left(r^{2}+s^{2}-2 r s t \geqslant a^{2}, r, s \in R X\right)$ by the assumption, we have $f(r, s, t) \geqslant h(r, s, t),(r, s \in R X, t \in[-1,1])$. Therefore, setting $w \equiv 1$, we have the following inequality by Theorem 2 ,

$$
P E_{f}(X, w) \geqslant \sum_{x, y \in X} g_{0}(\|x\|,\|y\|)-\sum_{x \in X} h(\|x\|,\|x\|, 1) .
$$

If $\sum_{x, y \in X} g_{0}(\|x\|,\|y\|)>\sum_{x \in X} h(\|x\|,\|x\|, 1)$, then the right hand side of (21) is positive, and so $P E_{f}(X, w)=+\infty$. Hence, there exist $x \neq y \in X$ such that $\|x-y\|^{2} \leqslant a^{2}$. This contradicts to that $X$ is a Euclidean $a$-code. 
Corollary 4.1. Let $X \subset \mathbb{R}^{d}$ be a Euclidean a-code, $R X=\left\{r_{1}, \ldots, r_{p}\right\}$ and $X_{i}=X \cap$ $r_{i} S^{d-1}$. Set $f_{i j}(r, s, t)=(r s)^{i} Q_{j}(t),(i, j \geqslant 0)$, and $h(r, s, t)=\sum_{i, j} a_{i j} f_{i, j}(r, s, t), a_{i j} \geqslant$ $0(\forall j \geqslant 1)$. Assume that $h(r, s, t) \leqslant 0,\left(r^{2}+s^{2}-2 r s t \geqslant a^{2}, r, s \in R X\right)$. Then the following inequality holds:

$$
\sum_{i} a_{i 0}\left(\sum_{k=1}^{p} r_{k}^{i}\left|X_{k}\right|\right)^{2} \leqslant \sum_{k=1}^{p} h\left(r_{k}, r_{k}, 1\right)\left|X_{k}\right| .
$$

Proof. Set $g_{i j}(r)=\sqrt{a_{i j}} r^{i}(j \geqslant 1)$ and $w \equiv 1$, and use Theorem 5 .

In (22), the left hand side is of degree two and the right hand side is of degree one with respect to $\left|X_{i}\right|$. So (22) gives an upper bound of $\left|X_{i}\right|$ if we could find a good function.

Remark 1. Let $X \subset \mathbb{R}^{d}$ be a Euclidean $a$-code and $\hat{x}:=\{\hat{x} \mid x \in X\}$. If $|\hat{x}|<|X|$, namely if there exist $x \neq y \in X$ such that $\hat{x}=\hat{y}$, then $X_{i}$ and $X_{j}$ are separated at least by $a$ where $x \in X_{i}$ and $y \in X_{j}$. Hence, the condition of a Euclidean $a$-code does not give any restriction between $X_{i}$ and $X_{j}$. Therefore, it is enough to consider the case $|\hat{x}|=|X|$. For $x, y \in X,(\|x\|=r,\|y\|=s)$, we have $\|x-y\|^{2}=r^{2}+s^{2}-2 r s t \geqslant a^{2}$. For $r, s \in R X$, set

$$
z_{r s}:=\frac{r^{2}+s^{2}-a^{2}}{2 r s}, z:=\max \left\{z_{r s} \mid r, s \in R X\right\} .
$$

Then since $X$ is a Euclidean $a$-code, we have $\hat{x} \cdot \hat{y} \leqslant z(\forall x, y \in X)$. So $\hat{x}$ is a spherical $z$-code. The linear programming bound of the usual Delsarte method for spherical $z$-codes is a method as seeking a polynomial $h(t)=\sum_{i} a_{i} Q_{i}(t)$ such that $a_{i} \geqslant 0(\forall i \geqslant 1), a_{0}>0$ and $h(t) \leqslant 0(\forall t \in[-1, z])$ (refer to [6]). In Corollary 4.1, if $a_{i j}=0(\forall i \geqslant 1)$, then $(22)$ is the same to the linear programming bound of the Delsarte method. Since functions $h(t)$ or bounds appearing in the Delsarte bounds are particular cases in ones of Corollary 4.1. So there is a possibility to improve the bound obtained by using the Delsarte method directly to Euclidean $a$-codes as above.

Example 1. Let $X$ be a Euclidean 1-code in $\mathbb{R}^{2}$ with radii 1 and 1.16 . We want to give an upper bound on $\left|X_{1}\right|$ and $\left|X_{2}\right|$. First we apply the usual Delsarte method to $X$. Under the same notation to Remark $1, z=1057 / 1682$. Set $a_{1}=1.013212587, a_{2}=0.6040849486$, $a_{3}=0.3325651473, a_{4}=0.1442439181, a_{5}=0.03803128022$ and

$$
h(t)=1+a_{1} Q_{1}(t)+a_{2} Q_{2}(t)+a_{3} Q_{3}(t)+a_{4} Q_{4}(t)+a_{5} Q_{5}(t) .
$$

$h(t)$ satisfies the conditions $a_{i} \geqslant 0(\forall i \geqslant 1), a_{0}>0$, and $h(t) \leqslant 0(\forall t \in[-1, z])$. Therefore by the Delsarte method, we obtain $|X|<h(1)=7.057164402$. This bound is the best one which is obtained by considering the projection $\hat{x}$ because the regular 7 -gon on the unit circle is a spherical $z$-code.

Next we apply Theorem 5. Let

$$
\begin{aligned}
& a_{10}=0.9880278352, a_{11}=0.9837793599, a_{20}=0.5699046690, a_{21}=1.335167172, \\
& a_{30}=0.2971650220, a_{31}=1.382817397, a_{40}=0.1180592309, a_{41}=1.022839342, \\
& a_{50}=0.02673442743, a_{51}=0.4412833120,
\end{aligned}
$$


and $g_{0}(r, s) \equiv 1, g_{i 1}(r)=\sqrt{a_{i 0}}, g_{i 2}(r)=\sqrt{a_{i 1}}(r-1)$ for $1 \leqslant i \leqslant 5$. Set

$$
h(r, s, t)=g_{0}(r, s)+\sum_{i=1}^{5}\left(g_{i 1}(r) g_{i 1}(s)+g_{i 2}(r) g_{i 2}(s)\right) Q_{i}(t) .
$$

Then we can check easily that $h(r, s, t)$ satisfies the conditions in Theorem 5 . Therefore by Theorem 5 , we have the following inequlity:

$$
|X|^{2} \leqslant\left|X_{1}\right| h(1,1,1)+\left|X_{2}\right| h(1.2,1.2,1)=6.625132485\left|X_{1}\right|+7.118347881\left|X_{2}\right|,
$$

where the second equality of the above is due to an approximate calculation. Solving the above inequality for each given $\left|X_{1}\right|$, we have that if $\left|X_{1}\right|=1$, then $\left|X_{2}\right| \leqslant 7-\left|X_{1}\right|$ and if $\left|X_{1}\right|=2,3,4,5$, then $\left|X_{2}\right| \leqslant 6-\left|X_{1}\right|$. This implies that Theorem 5 improves the Delsarte method in the case when $\left|X_{1}\right|=2,3,4,5$.

Now we give a linear programming bound on the cardinality of a Euclidean $t$-design. Set $A\left(X_{(r)}, X_{(s)}\right):=\left\{\hat{x} \cdot \hat{y} \mid x \in X_{(r)}, y \in X_{(s)}, \hat{x} \neq \hat{y}\right\}$.

Theorem 6. Let $(X, w)$ be a Euclidean t-design. Suppose that $w(x)$ is constant on each concentric sphere and denote by $w(\|x\|):=w(x)$. Set

$$
I:=\left\{(i, j) \in \mathbb{Z}_{\geqslant 0}^{2} \mid 0 \leqslant i \leqslant t \text {, there exists } k \in \mathbb{Z}_{\geqslant 0} \text { such that } i=2 k+j \text { or } j=0\right\} .
$$

Assume that for any $(i, j) \notin I, a_{i j} \leqslant 0$ and $f(r, s, t)=\sum_{i, j \geqslant 0} a_{i j} f_{i j}(r, s, t)$. Moreover, assume that $f(r, s, t) \geqslant 0(\forall r, s \in R X, \forall t \in[-1,1])$. Then, we have the following inequality:

$$
\sum_{i \geqslant 0} a_{i 0}\left(\sum_{r \in R X} w(r) r^{i}\left|X_{(r)}\right|\right)^{2} \geqslant \sum_{r, s \in R(X)} w(r) w(s) f(r, s, 1) d_{r, s, 1}
$$

where

$$
d_{r, s, t}:=\sharp\left\{(x, y) \in X^{2} \mid x \in X_{(r)}, y \in X_{(s)}, \hat{x} \cdot \hat{y}=t\right\} .
$$

Proof. We estimate the following value:

$$
\sum_{i, j} a_{i j} \sum_{x, y \in X} w(x) w(y) f_{i j}(\|x\|,\|y\|, \hat{x} \cdot \hat{y})
$$

Since $(X, w)$ is a Euclidean $t$-design,

$$
\begin{aligned}
(24) & =\sum_{i \geqslant 0} a_{i 0} \sum_{x, y \in X} w(x) w(y)(\|x\|\|y\|)^{i} \\
& +\sum_{(i, j) \notin I} a_{i j} \sum_{x, y \in X} w(x) w(y)(\|x\|\|y\|)^{i} Q_{j}(\hat{x} \cdot \hat{y}) .
\end{aligned}
$$


By the assumption, $a_{i j} \leqslant 0(\forall(i, j) \notin I)$ and by Lemma 2.4 ,

$$
\sum_{x, y \in X} w(x) w(y)(\|x\|\|y\|)^{i} Q_{j}(\hat{x} \cdot \hat{y}) \geqslant 0 .
$$

Hence,

$$
(24) \leqslant \sum_{i \geqslant 0} a_{i 0} \sum_{x, y \in X} w(x) w(y)(\|x\|\|y\|)^{i}=\sum_{i \geqslant 0} a_{i 0}\left(\sum_{r \in R X} w(r) r^{i}\left|X_{(r)}\right|\right)^{2} .
$$

On the other hand, by $f(r, s, t) \geqslant 0(\forall r, s \in R X, \forall t \in[-1,1])$, we have

$$
\begin{aligned}
(24) & =\sum_{x, y \in X} w(x) w(y) \sum_{i, j} a_{i j} f_{i j}(\|x\|,\|y\|, \hat{x} \cdot \hat{y}) \\
& =\sum_{x, y \in X} w(x) w(y) f(\|x\|,\|y\|, \hat{x} \cdot \hat{y}) \\
& \geqslant \sum_{r, s \in R(X)} w(r) w(s) f(r, s, 1) d_{r, s, 1}
\end{aligned}
$$

In (23), the left hand side is of degree two and the right hand side is of degree one with respect to $\left|X_{i}\right|$. Therefore (23) gives a lower bound of $\left|X_{i}\right|$ if we could find a good function $f$.

Acknowledgements The authors would like to thank Professors Eiichi Bannai, Motoko Kotani, Akihiro Munemasa for the helpful discussions and comments.

\section{References}

[1] Ei. Bannai and Et. Bannai, Algebraic Combinatorics on Spheres (in Japanese) Springer Tokyo 1999.

[2] Ei. Bannai, Et. Bannai, M. Hirao and M. Sawa, Cubature formulas in numerical analysis and Euclidean tight designs, European J. Combin. 31 (2010), 423-441.

[3] Et. Bannai, On antipodal Euclidean tight $(2 e+1)$-designs, J. Algebraic Combin. 24 (2006), 391-414.

[4] Et. Bannai, More about Euclidean designs, Finite Groups and Algebraic Combinatorics (Japanese) (Kyoto, 2007), Sūrikaisekikenkyūsho Kōkyūroku 1593 (2008), $113-121$.

[5] H. Cohn and A. Kumar, Universally optimal distribution of points on spheres, J. Amer. Math. Soc. 20 (2007), no. 1, 99-148.

[6] P. Delsarte, Bounds for unrestricted codes, by linear programming, Philips Res. Rept, 27 (1972), 272-289. 
[7] P. Delsarte and J. J. Seidel, Fisher type inequalities for Euclidean $t$-designs, Lin. Algebra and its Appl. 114-115 (1989), 213-230.

[8] P. Delsarte, J. M. Goethals and J. J. Seidel, Spherical codes and designs, Geom. Dedicata 6 (1977), 363-388.

[9] H. M. Möller, Kubaturformeln mit minimaler Knotenzahl, Nuemer. Math. 25 (2) (1975/76), 185-200.

[10] H. M. Möller, Lower bounds for the number of nodes in cubature formulae, $\mathrm{Nu}$ merische Integration (Tagung, Math. Forschungsinst., Oberwolfach, 1978), 221-230, Internat. Ser. Numer. Math., 45, Birkhhöuser, Basel-Boston, Mass., 1979.

[11] A. Neumaier and J. J. Seidel, Discrete measures for spherical designs, eutactic stars and lattices, Nederl. Akad. Westensch. Proc. Ser. A 91 (Indag. Math. 50) (1988), 321-334.

[12] I. J. Schoenberg, Positive definite functions on spheres, Duke Math. J. 9 (1942), 96-108.

[13] P. Seymour and T. Zaslavsky, Averaging sets: a generalization of mean values and spherical designs, Adv. in Math. 52 (1984), no. 3, 213-240.

[14] V.M. Sidelnikov, New bounds for the density of sphere packings in an $n$-dimensional Euclidean space, Mat. Sb. 85 (1974), English translation, Math. USSR Sbornik, 24 (1974), 147-157.

[15] V. A. Yudin, Minimum potential energy of a point system of charges (Russian), Diskret. Mat. 4 (1992), 115-121; translation in Discrete Math. Appl. 3 (1993), 7581. 